\title{
On the Numerical Evaluation of Two Infinite Products
}

\author{
By G. Allasia and F. Bonardo
}

\begin{abstract}
A numerical evaluation of two infinite products of the type $\Pi_{n=0}^{\infty}\left(1-a q^{n}\right)$, which are important in some mathematical fields, is considered.

The numerical evaluation is based on a recursive formula of the type $x_{n+1}=$ $x_{n} f\left(y_{n} / x_{n}\right), y_{n+1}=x_{n+1} g\left(y_{n} / x_{n}\right)$, and it is compared with a series expansion that was previously used for the computation.

Two tables of the infinite products are provided with twenty significant figures which check and extend existing data.
\end{abstract}

1. Introduction. The infinite product

$$
P=\prod_{n=0}^{\infty}\left(1-a q^{n}\right), \quad|a|<1, \quad 0 \leqslant q<1,
$$

and its particular case, for $a=q$,

$$
Q=\prod_{n=1}^{\infty}\left(1-q^{n}\right)
$$

are very important in some mathematical fields (elliptic, hypergeometric, modular and partition functions); and therefore, it is very useful to have their numerical evaluation.

A table of the reciprocal of (1.1) has been built by L. Slater [1], [2] with seven significant figures for $a=-0.90(0.05) 0.95$ and $q=0.00(0.05) 0.95$. The table was constructed using the formula

$$
P^{-1}=\sum_{n=0}^{\infty} \frac{a^{n}}{(q)_{n}},
$$

where

$$
(q)_{n}=(1-q)\left(1-q^{2}\right) \cdots\left(1-q^{n}\right), \quad(q)_{0}=1 .
$$

L. Slater mentions some computing difficulties for $0.89<q<1$ that can be avoided by using the logarithmic form:

$$
\ln P=\ln \prod_{n=0}^{\infty}\left(1-a q^{n}\right)=\sum_{n=0}^{\infty} \ln \left(1-a q^{n}\right) .
$$

Except for the tables in [3] and a short table in [4], we know of no other tables.

Received August 20, 1979.

1980 Mathematics Subject Classification. Primary 65D20. 
2. The Algorithm. L. Gatteschi has suggested [5] the iterative scheme

$$
\begin{aligned}
& x_{n+1}=x_{n} \frac{(q-1) x_{n}+(3-q) y_{n}}{2 y_{n}}, \\
& y_{n+1}=x_{n+1} \frac{y_{n}}{q x_{n}+(1-q) y_{n}},
\end{aligned}
$$

If the initial values $\left(x_{0}, y_{0}\right)$ satisfy the constraint

$$
\frac{x_{0}}{y_{0}}=\frac{1+2 a-q}{1-q},
$$

then the algorithm (2.1) has the limit function

$$
X\left(x_{0}, y_{0}\right)=\lim _{n \rightarrow \infty} x_{n}=\lim _{n \rightarrow \infty} y_{n}=x_{0} \cdot P
$$

provided $x_{0} \neq 0$, that is $1+2 a-q \neq 0$. In the case $x_{0}=0$, that is, $a=(q-1) / 2$, the infinite product $(1.1)$ can be replaced by

$$
\prod_{n=0}^{\infty}\left(1-a^{*} q^{n}\right)=\frac{P}{1-a},
$$

where $a^{*}=a q$. In this way we have

$$
1+2 a^{*}-q=(q-1)^{2} \neq 0,
$$

and the given procedure applies.

A neat property of algorithm (2.1) is its bilateral convergence; that is, for appropriate initial values $x_{0}$ and $y_{0},\left\{x_{n}\right\}$ and $\left\{y_{n}\right\}$ are upper and lower bounds, respectively, of the limit (2.2). Thus, the sequence $\left\{t_{n}\right\}$,

$$
t_{n}=1 / 2\left(x_{n}+y_{n}\right)
$$

is an improved approximation over $x_{n}$ and $y_{n}$.

3. Related Infinite Products. There are some cases that can be reduced to the form (1.1) and, therefore, computed with the algorithm (2.1). They are

$$
\begin{gathered}
\prod_{n=1}^{\infty}\left(1+q^{n}\right), \\
\prod_{n=1}^{\infty}\left(1-q^{2 n}\right)=Q_{0}, \\
\prod_{n=1}^{\infty}\left(1+q^{2 n}\right)=Q_{1}, \\
\prod_{n=1}^{\infty}\left(1+q^{2 n-1}\right)=Q_{2}, \\
\prod_{n=1}^{\infty}\left(1-q^{2 n-1}\right)=Q_{3} .
\end{gathered}
$$

The infinite product (3.1) is obtained from (1.1) by setting $a=-q ;(3.2)$ and (3.3) can be reduced to (1.2) and (3.1), respectively, with the substitution $q^{2}=p$; the infinite products (3.4) and (3.5) can be reduced to (1.1) by putting $q^{2}=p$ and $a=$ $\mp q$. 
The infinite products (3.1) to (3.4) are related [7] since

$$
Q_{0} Q_{3}=\prod_{n=1}^{\infty}\left(1-q^{n}\right), \quad Q_{1} Q_{2}=\prod_{n=1}^{\infty}\left(1+q^{n}\right), \quad Q_{1} Q_{2} Q_{3}=1
$$

A table of $\ln Q_{0}$ has been published by F. W. Newman [3], [6]. This table has sixteen significant figures and has been computed for $\rho=-1 / 2 \ln q=1.0(0.1) 4.6$.

4. Numerical Evaluation. The computation was done on the ELEA 6001 computer at the Institute of Numerical Analysis of the University of Turin. To avoid the loss of significant digits we used a floating-point arithmetic package with a twentythree-digit mantissa.

The values given in Table 1 were found using both (1.3) and (2.1), and so may be considered exact to the number of places given.

Regarding the particular case (1.2), L. Slater published [1] , [2] a table of numerical values of its reciprocal for $q=0.000(0.005) 0.995$ with seven significant figures. We extended this table to twenty significant figures (unrounded) using (1.3) and (2.1) (Table 2).

As noted earlier, L. Slater reported difficulties in computing with (1.3) for 0.89 $<q<1$ in view of slow convergence. For this range, we would suggest the algorithm [8]

$$
\begin{aligned}
& z_{n+1}=q z_{n}, \quad z_{0}=a, \\
& w_{n+1}=w_{n}\left(1+z_{n}\right), \quad w_{0}=1 .
\end{aligned}
$$

Indeed we computed Table 1 in the above range using the latter scheme. Though convergence is slow, the algorithm is very simple and easy to do on a computer.

TABLE 1*

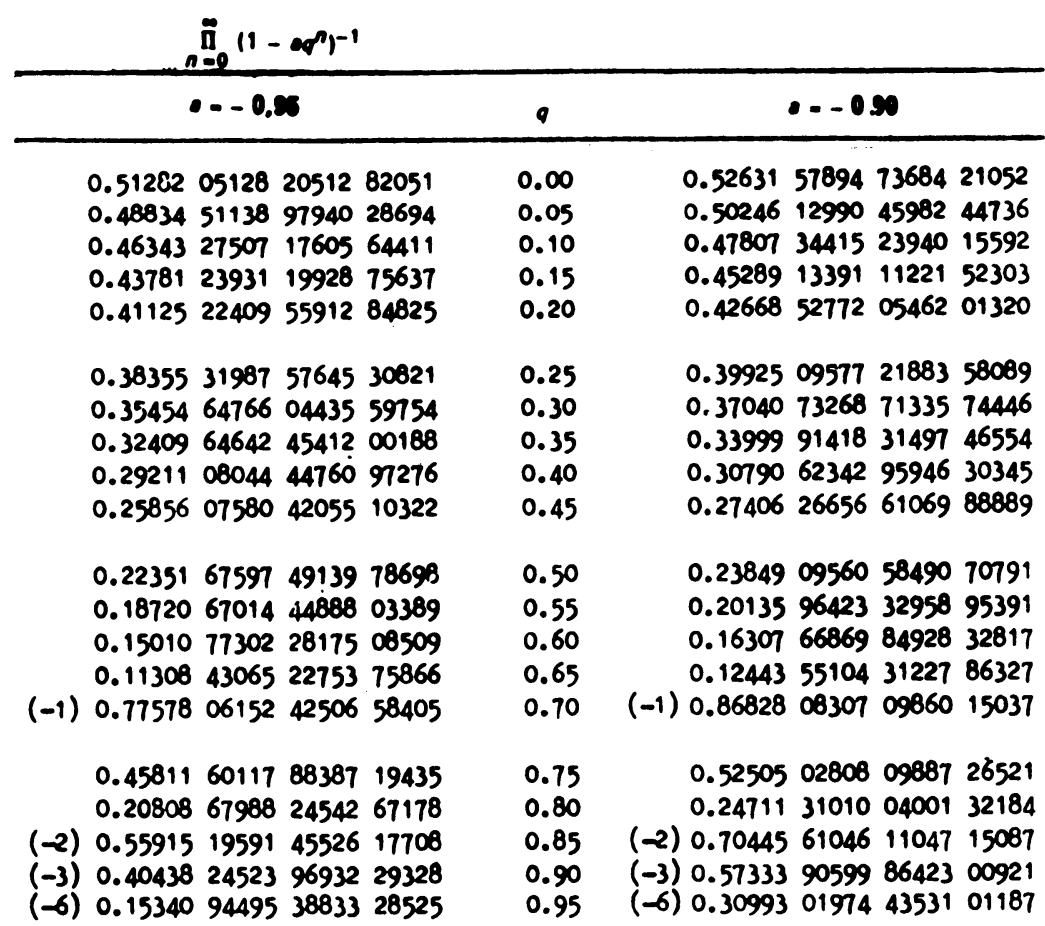

* The notation $(k) 0 . n$ means $0 . n 10^{k}$. The number $(k)$ is omitted after its first appearance. 
TABLE 1 (continued)

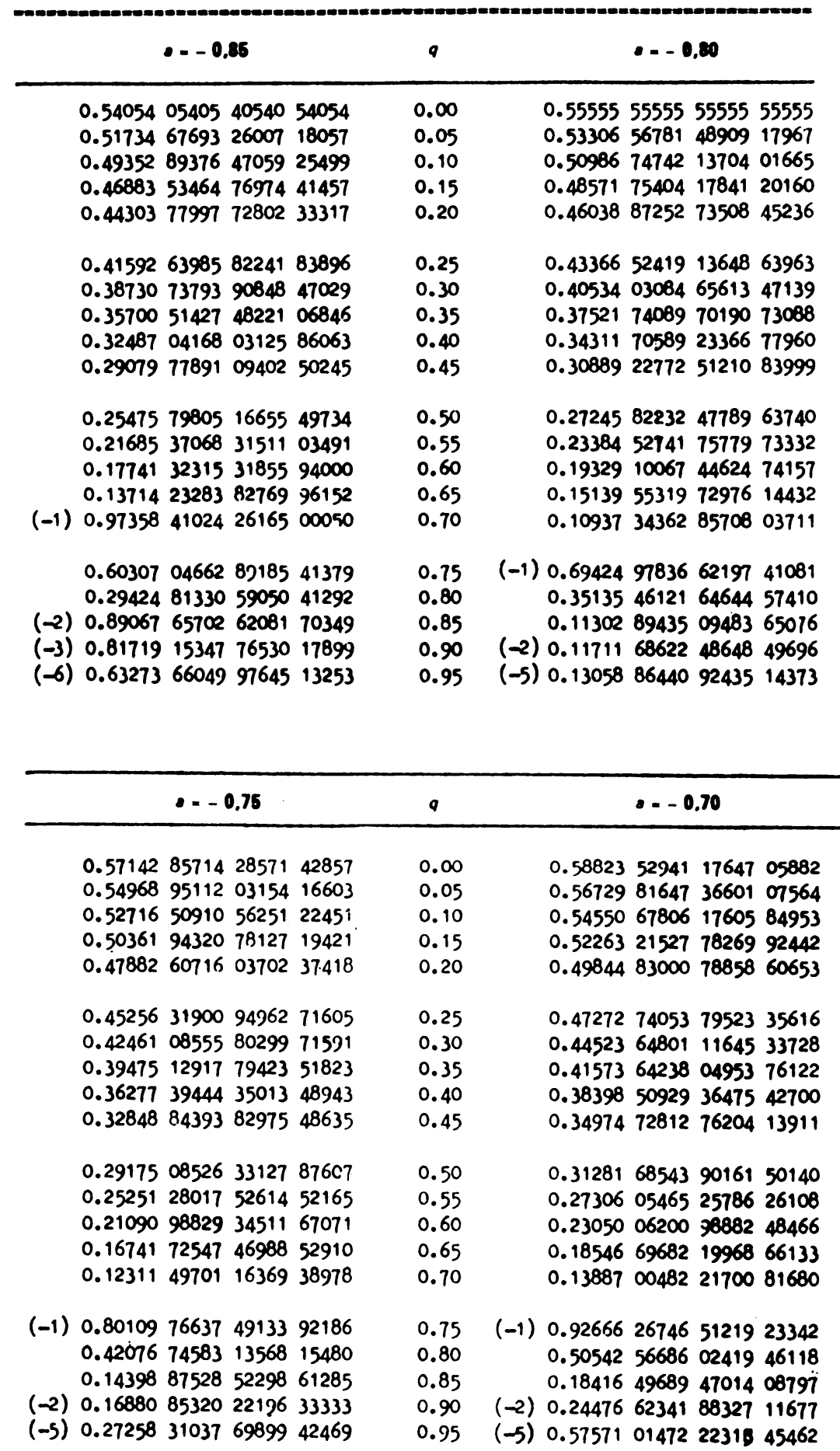


TABLE 1 (continued)

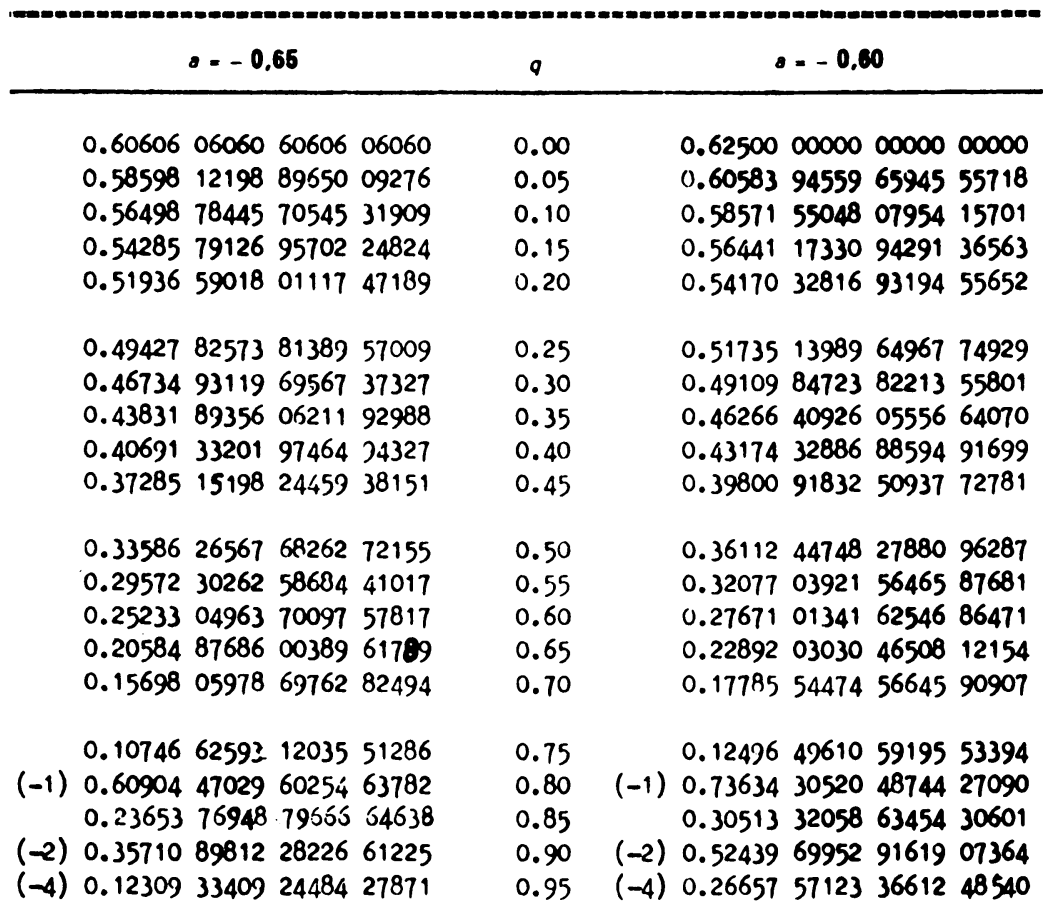

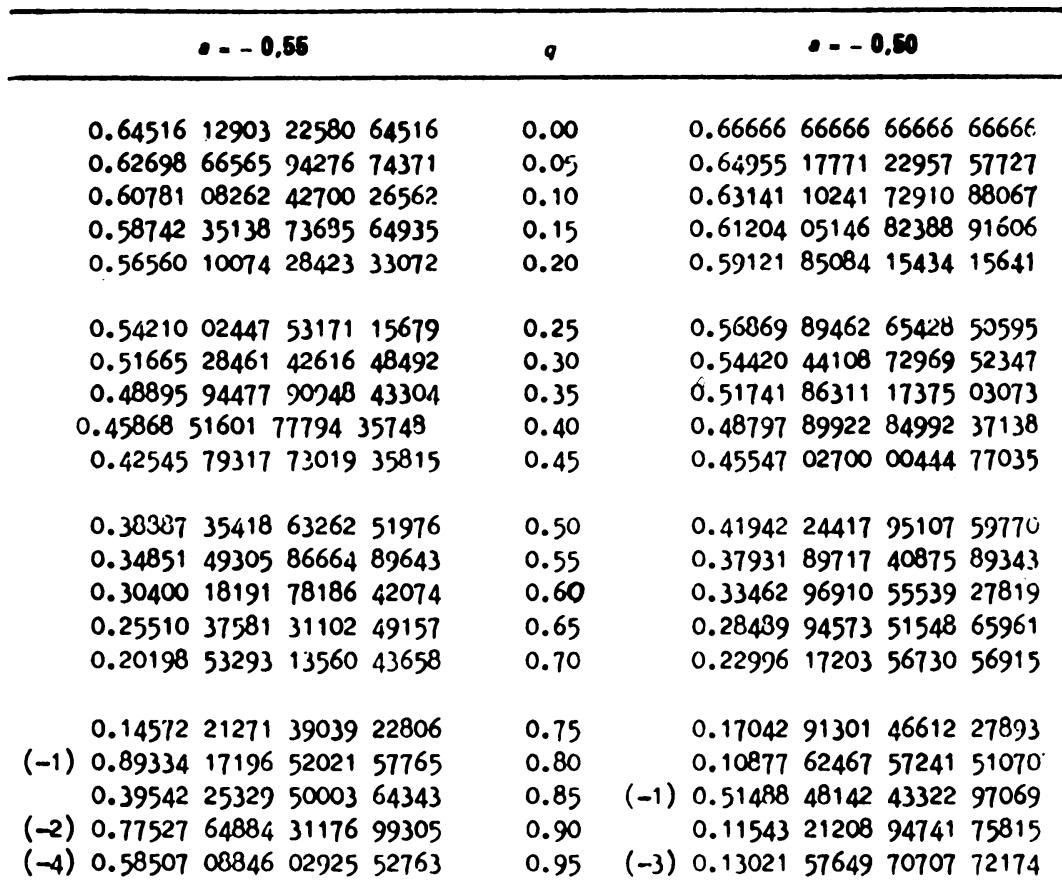


TABLE 1 (continued)

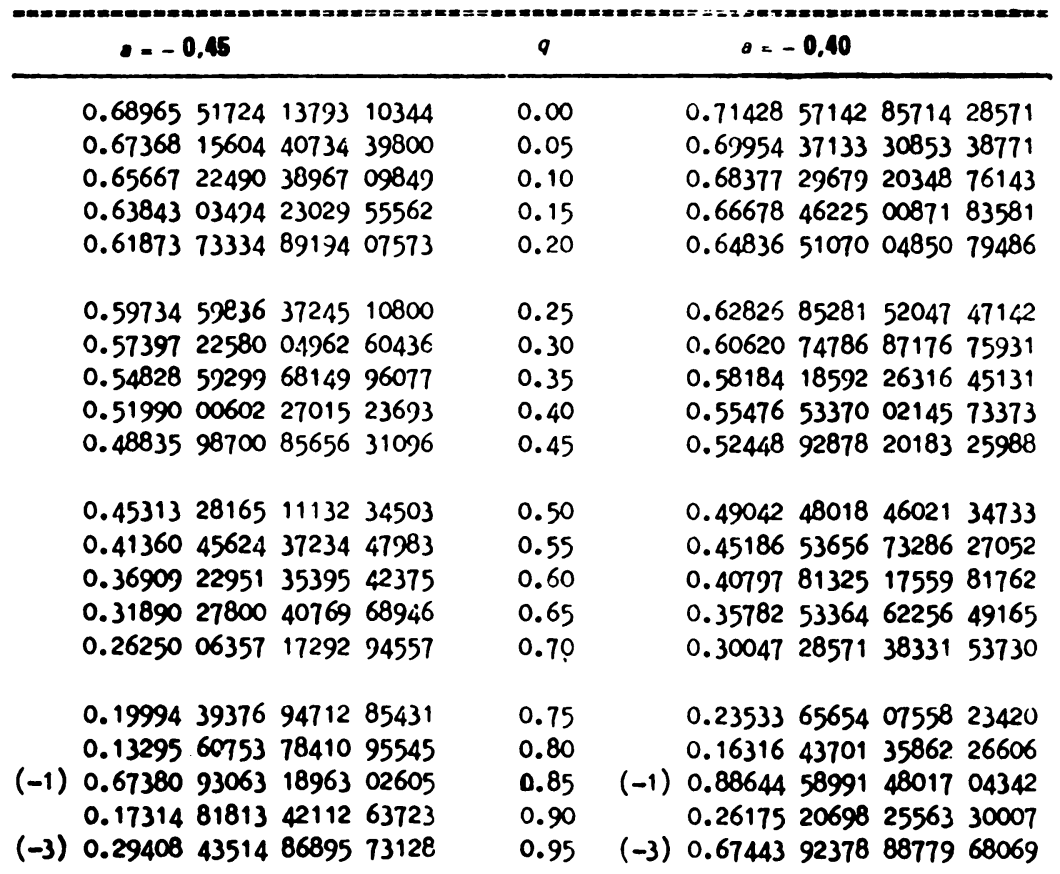

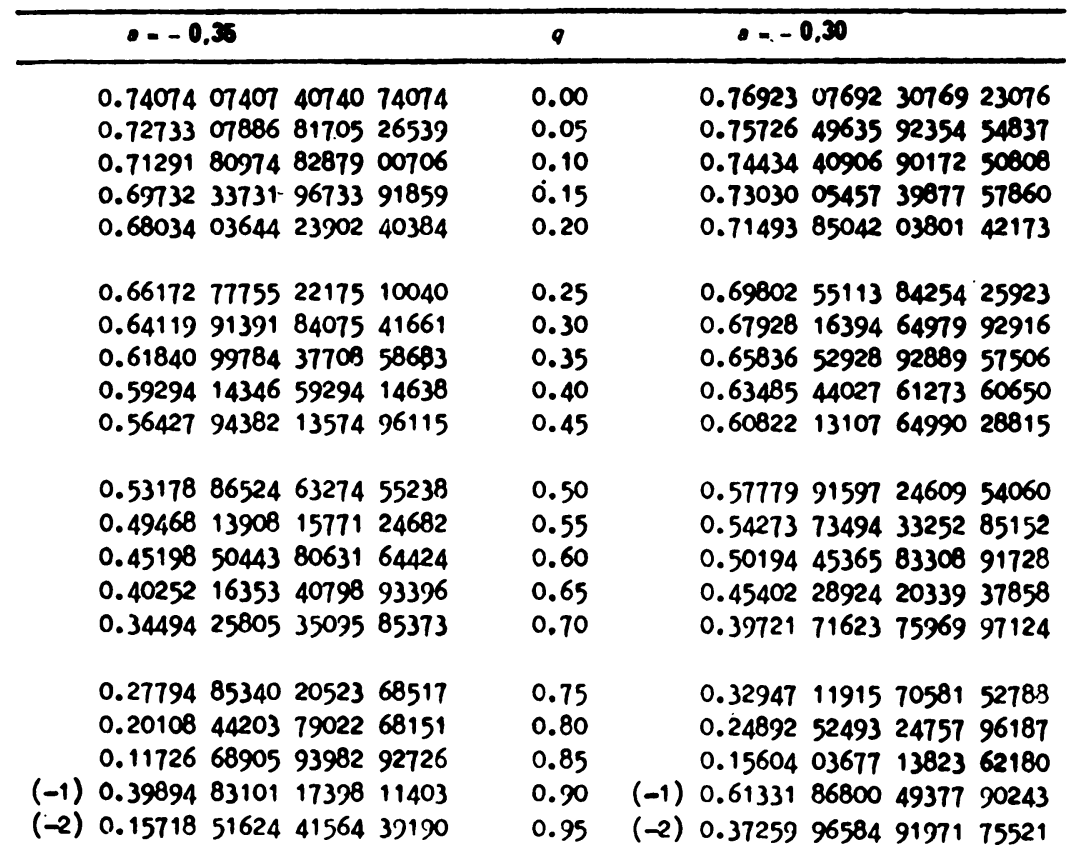


TABLE 1 (continued)

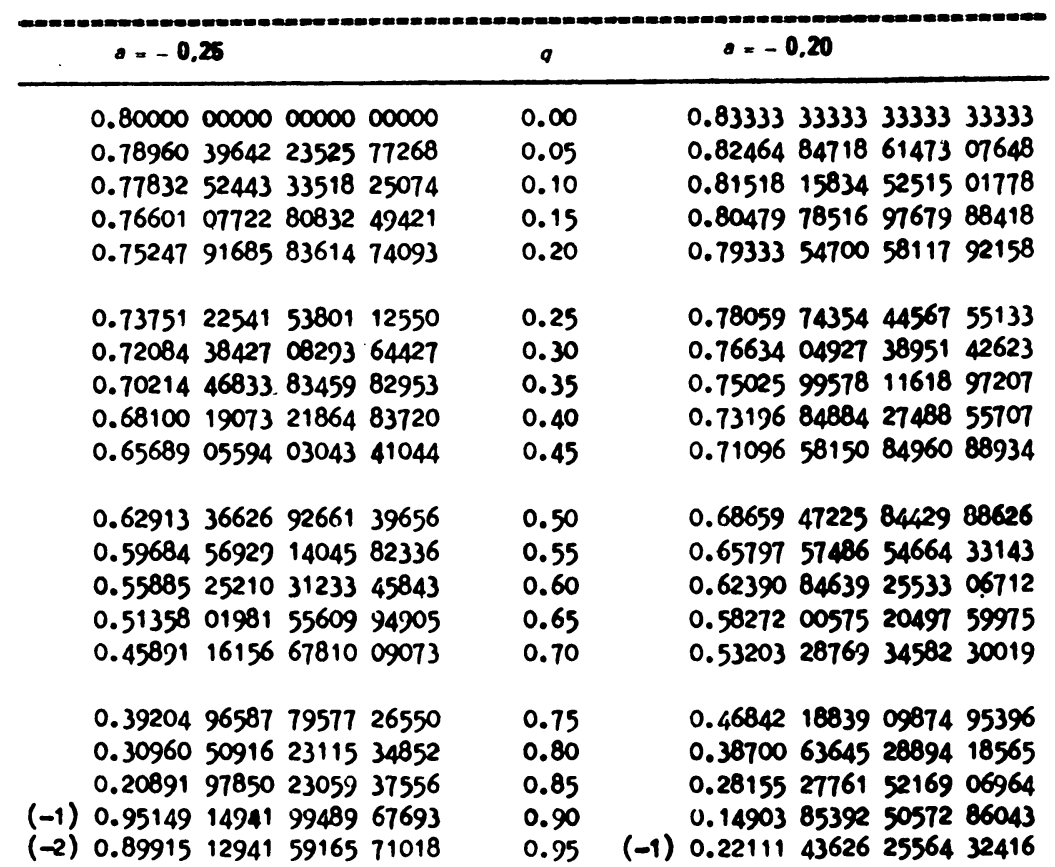

\begin{tabular}{|c|c|c|}
\hline-0.0 .15 & 9 & $\cdot=-0,10$ \\
\hline $\begin{array}{llll}0.86956 & 52173 & 91304 & 34782 \\
0.86275 & 14609 & 98656 & 78227 \\
0.85528 & 88025 & 70030 & 14144 \\
0.84706 & 54403 & 63857 & 76920 \\
0.83794 & 56253 & 06479 & 15481\end{array}$ & $\begin{array}{l}0.00 \\
0.05 \\
0.10 \\
0.15 \\
0.20\end{array}$ & $\begin{array}{llll}0.90909 & 09090 & 90909 & 09090 \\
0.90433 & 00841 & 63850 & 03301 \\
0.89909 & 09181 & 82726 & 45446 \\
0.89329 & 06586 & 88780 & 71214 \\
0.88682 & 77626 & 23151 & 23462\end{array}$ \\
\hline $\begin{array}{lllll}0.82776 & 22383 & 43948 & 39887 \\
0.81630 & 66163 & 09454 & 28521 \\
0.80331 & 43243 & 60769 & 15525 \\
0.78844 & 48382 & 26206 & 71701 \\
0.77125 & 18656 & 84458 & 66041\end{array}$ & $\begin{array}{l}0.25 \\
0.30 \\
0.35 \\
0.40 \\
0.45\end{array}$ & $\begin{array}{llll}0.87957 & 59394 & 12866 & 45999 \\
0.87137 & 58606 & 27797 & 62083 \\
0.86202 & 33769 & 85695 & 12190 \\
0.85125 & 23841 & 52331 & 04651 \\
0.83870 & 92661 & 30010 & 47325\end{array}$ \\
\hline $\begin{array}{llll}0.75113 & 89076 & 41992 & 40278 \\
0.72729 & 08754 & 37917 & 55544 \\
0.69856 & 56512 & 89041 & 82304 \\
0.66331 & 64368 & 84673 & 18503 \\
0.61909 & 08216 & 88361 & 11764\end{array}$ & $\begin{array}{l}0.50 \\
0.55 \\
0.60 \\
0.65 \\
0.70\end{array}$ & $\begin{array}{llll}0.82391 & 36671 & 01315 & 86352 \\
0.80619 & 62712 & 98887 & 56967 \\
0.78459 & 53047 & 20531 & 19851 \\
0.75768 & 50559 & 08643 & 69290 \\
0.72324 & 58313 & 78623 & 05316\end{array}$ \\
\hline $\begin{array}{llll}0.56210 & 62606 & 31849 & 94475 \\
0.48633 & 32200 & 81862 & 84878 \\
0.38207 & 63939 & 81564 & 92875 \\
0.23583 & 89323 & 20549 & 08249 \\
0.55471 & 73896 & 369 ! \beta & 02345\end{array}$ & $\begin{array}{l}0.75 \\
0.80 \\
0.85 \\
0.90 \\
0.95\end{array}$ & $\begin{array}{llll}0.67765 & 71018 & 53959 & 00416 \\
0.61461 & 78461 & 85306 & 09883 \\
0.52232 & 59130 & 74359 & 35772 \\
0.37725 & 04151 & 70770 & 49557 \\
0.14214 & 20228 & 60639 & 77132\end{array}$ \\
\hline
\end{tabular}


TABLE 1 (continued)

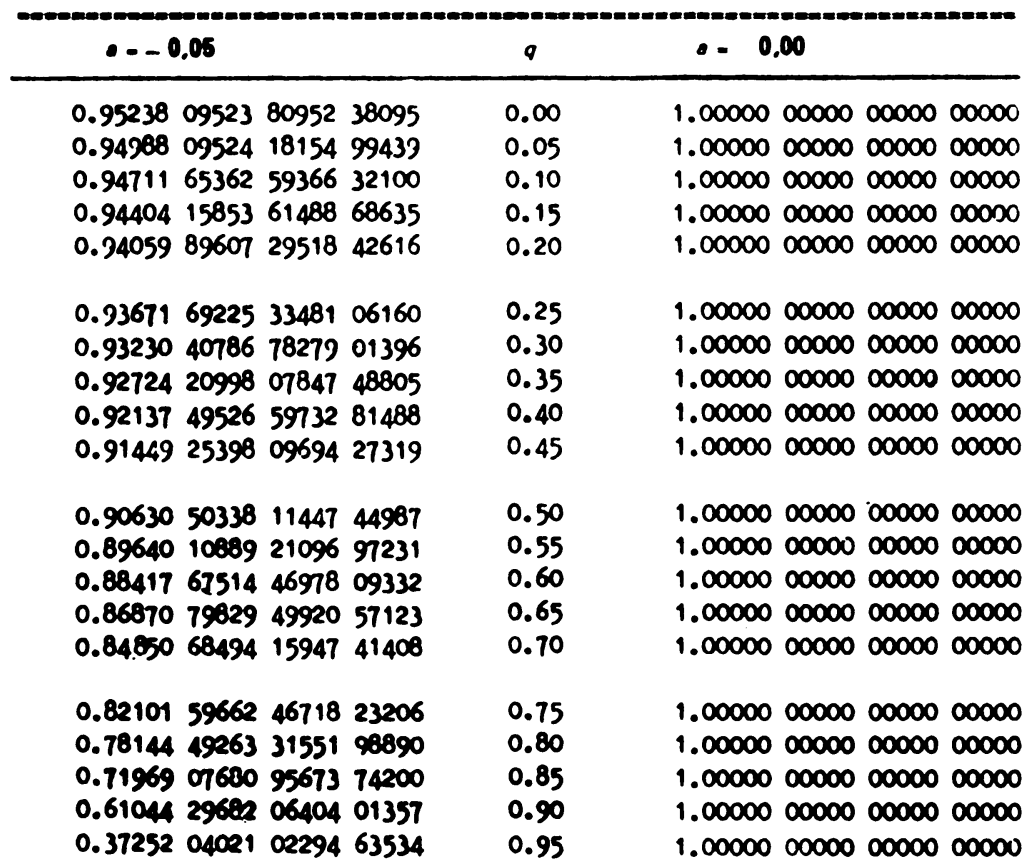

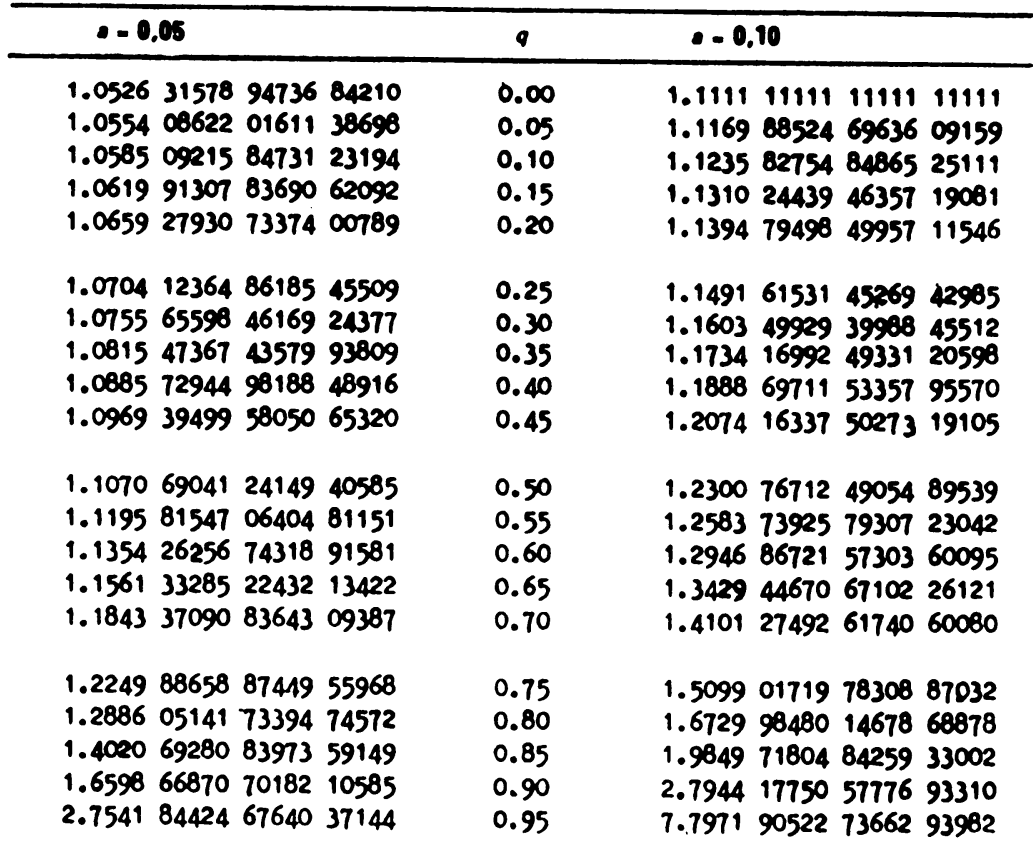


TABLE 1 (continued)

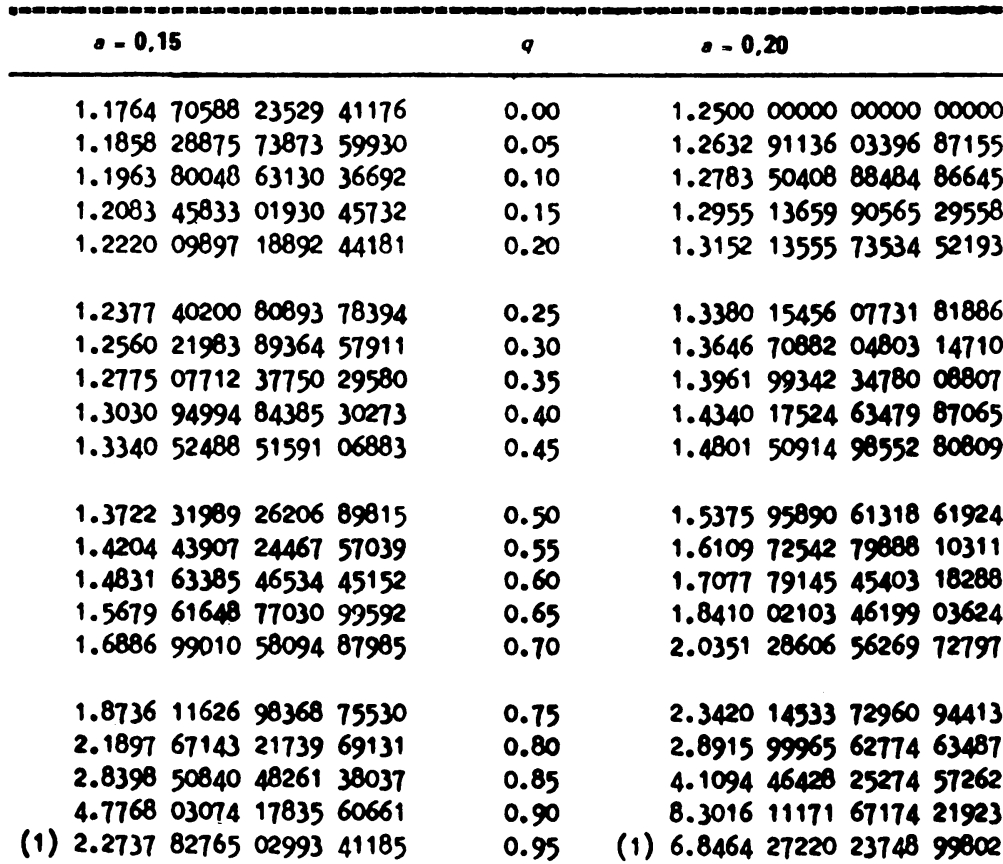

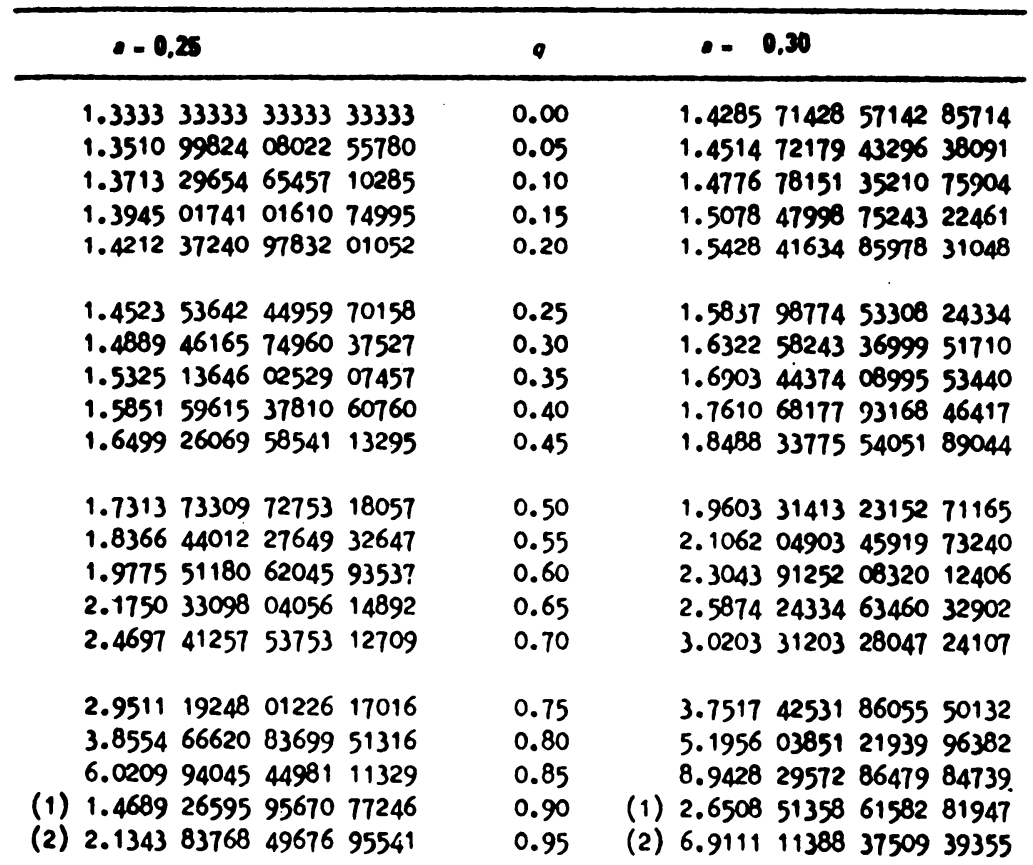


TABLE 1 (continued)

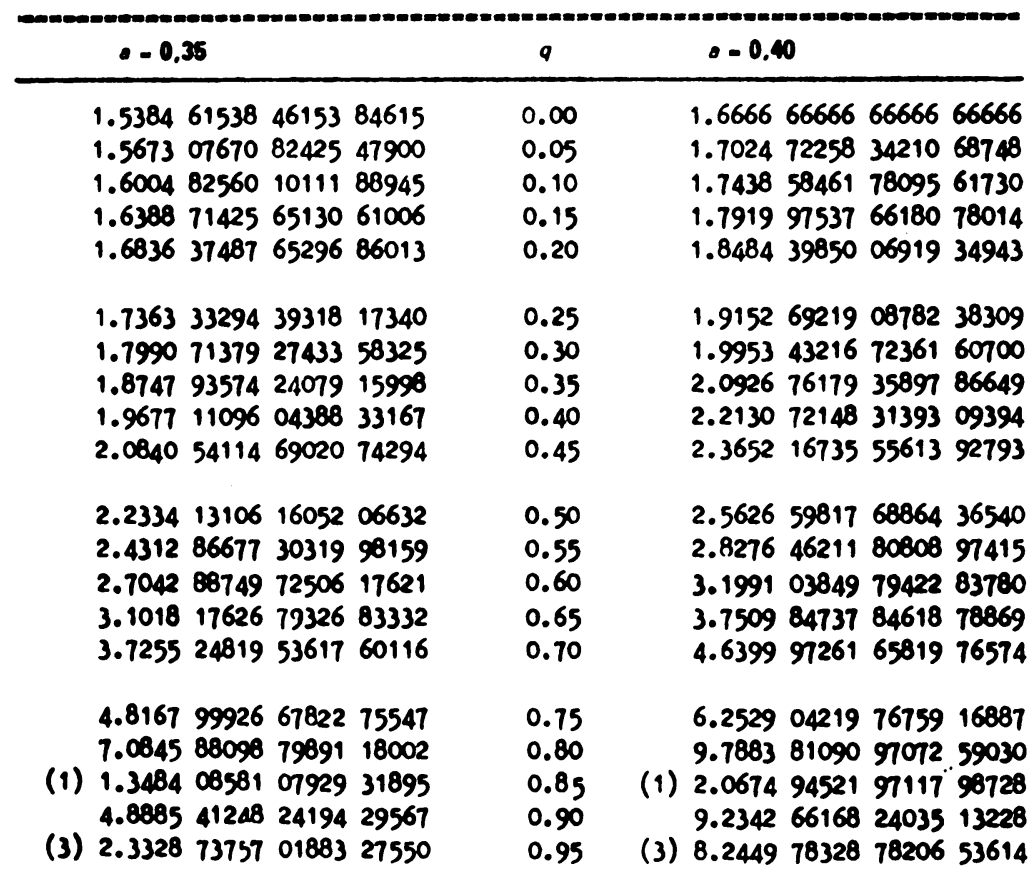

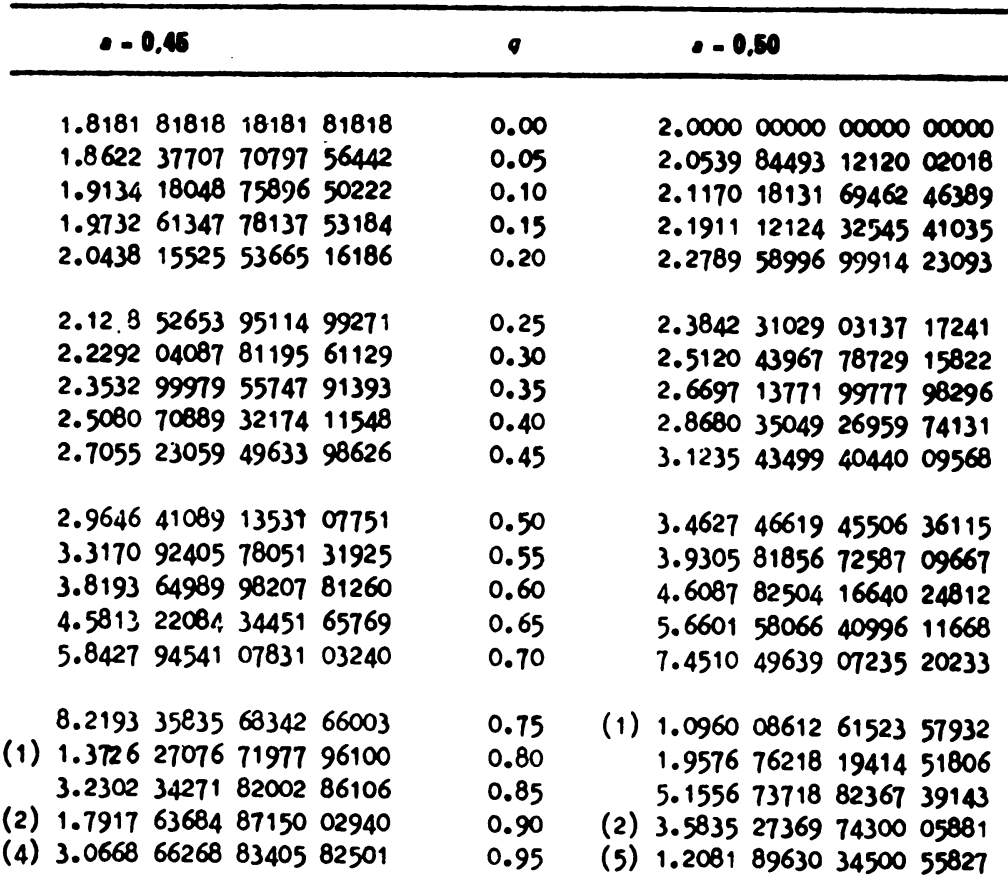


TABLE 1 (continued)

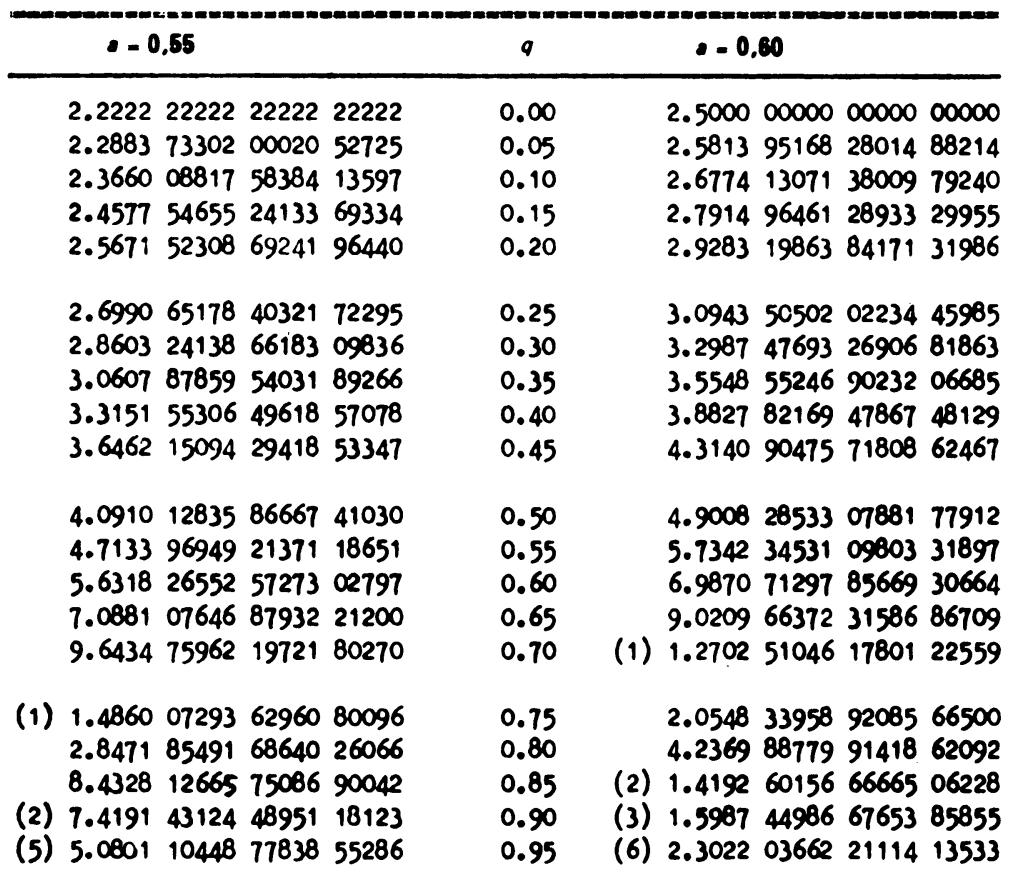

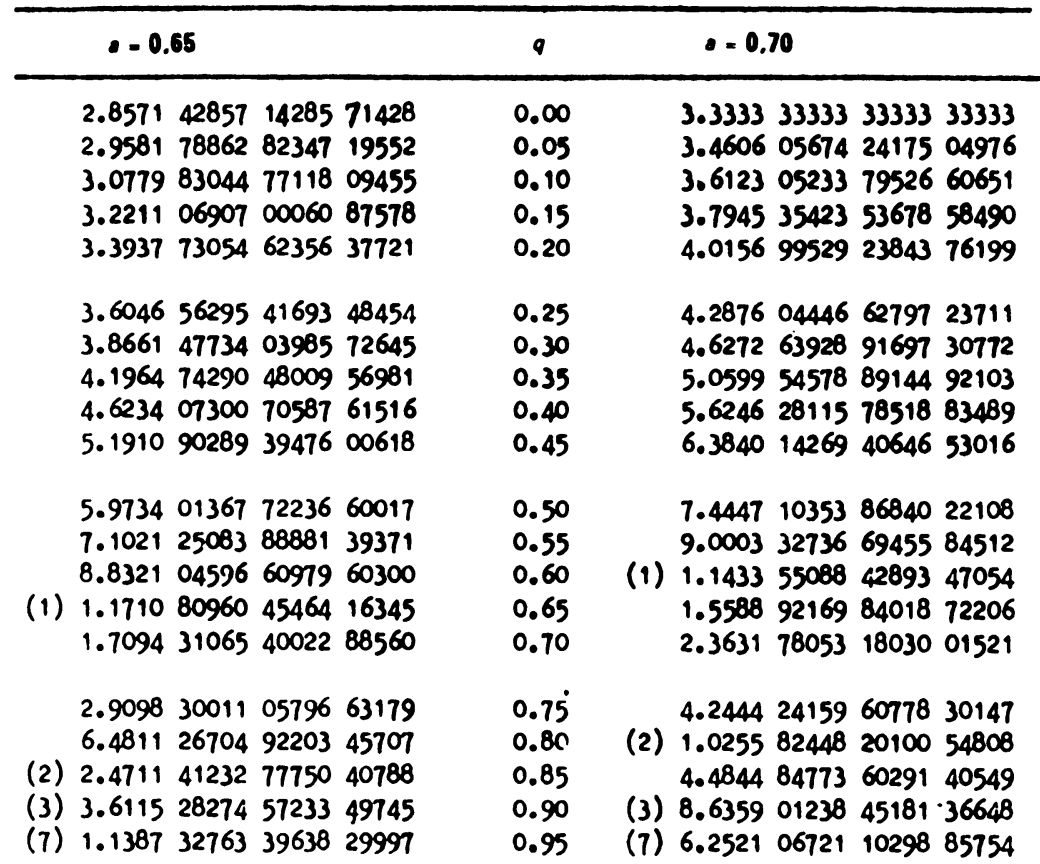


TABLE 1 (continued)

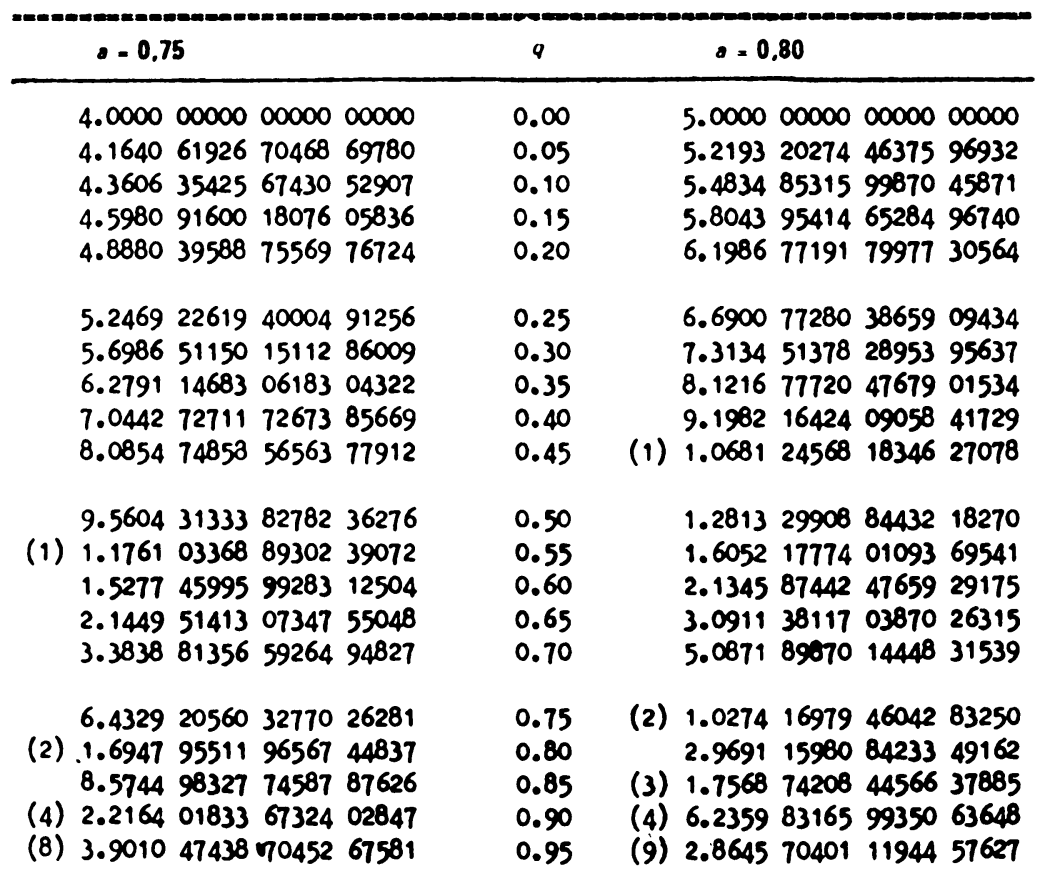

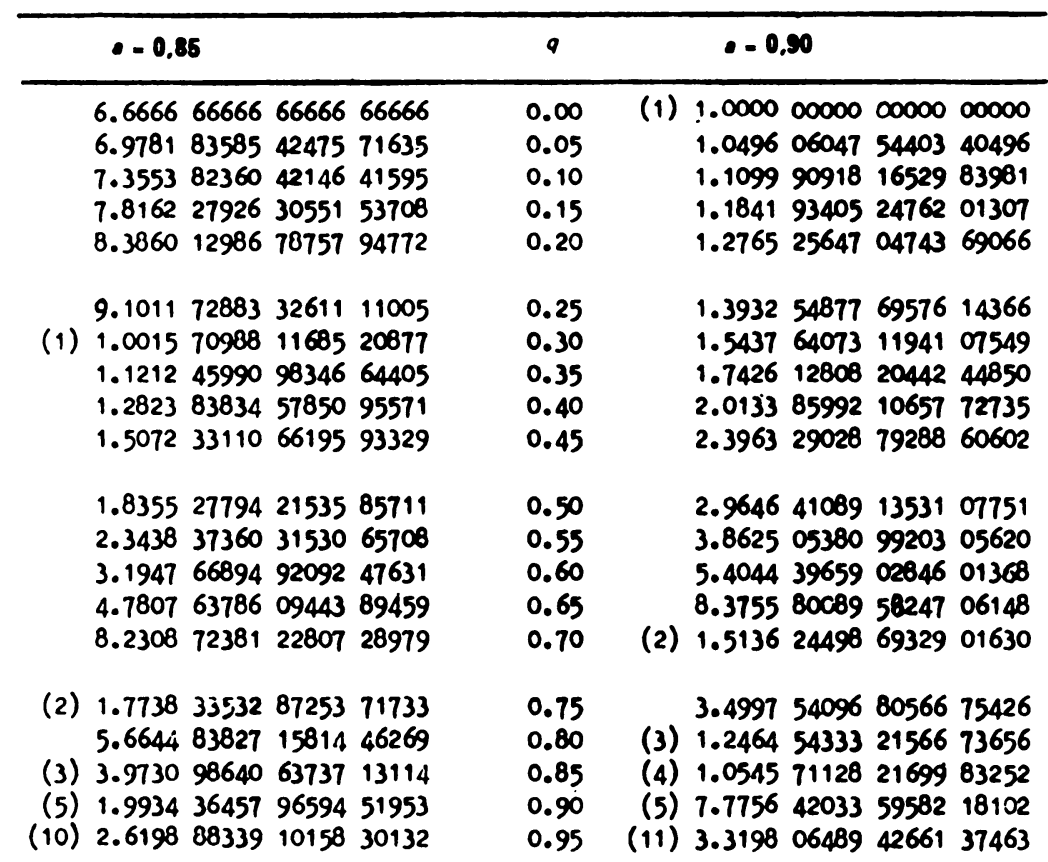


TABLE 1 (continued)

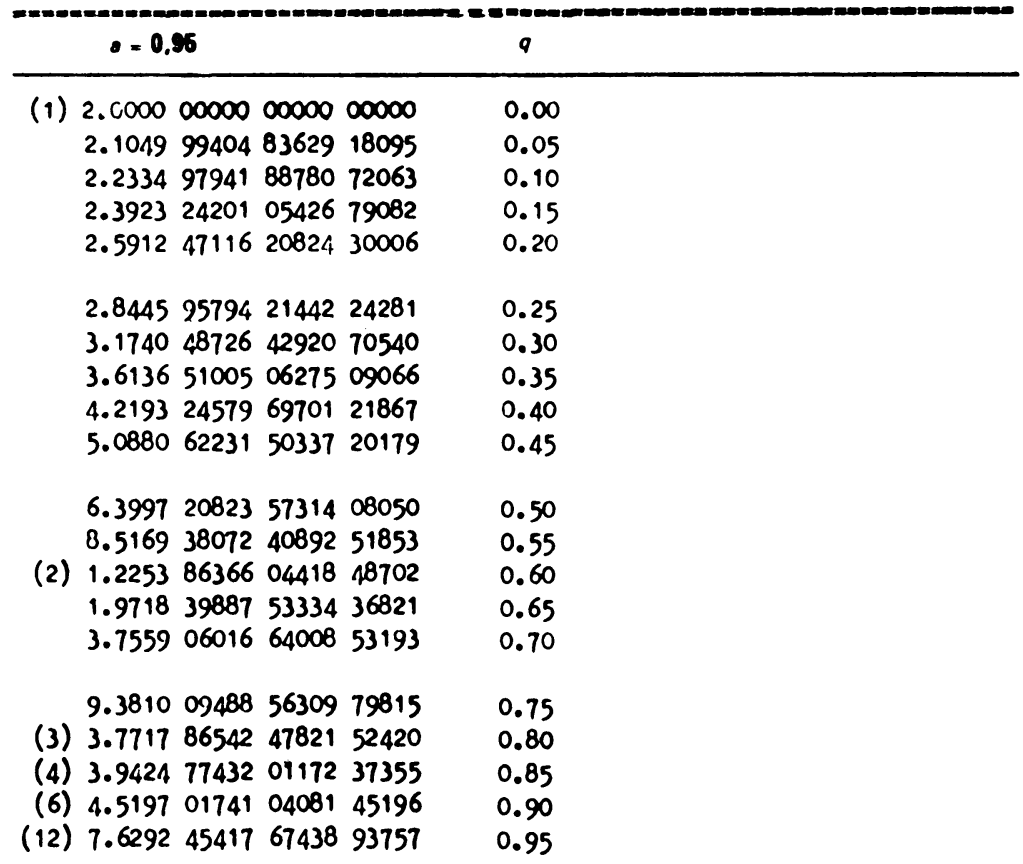

TABLE 2

$\prod_{n=1}^{\infty}(1-\infty)^{-1}$

\begin{tabular}{|c|c|c|c|}
\hline a & & a & \\
\hline 0.000 & 1.0000000000000000000 & 0.200 & $1.3152 \quad 135357353452193$ \\
\hline 0.005 & 1.0050503781470480555 & 0.205 & 1.3274002784150594186 \\
\hline 0.010 & 1.0102030507111522304 & 0.210 & 1.3396962998348283681 \\
\hline 0.015 & 1.0154603835685423272 & 0.215 & $1.3527 \quad 120565457020490$ \\
\hline 0.020 & 1.0208248231237790018 & 0.220 & 1.3658584406377814523 \\
\hline 0.025 & 1.0262988992649501275 & 0.225 & 1.3793468237170950330 \\
\hline 0.030 & $1.03188522846210 \quad 05252$ & 0.230 & 1.3931890823685356213 \\
\hline 0.035 & 1.0375865170161635943 & 0.235 & 1.4073976252103111654 \\
\hline 0.040 & 1.0434055644661087734 & 0.240 & 1.4219854216538149656 \\
\hline 0.045 & $1.049345267 \quad 1624934265$ & 0.245 & 1.4369660324920275251 \\
\hline 0.050 & 1.0554086220161138698 & 0.250 & $1.452353642 \quad 4495970158$ \\
\hline 0.055 & 1.0615907304309901598 & 0.255 & 1.4681630948386908490 \\
\hline 0.060 & $1.0679 \quad 18802 \quad 4314954887$ & 0.260 & 1.4844099284766580064 \\
\hline 0.065 & 1.0743721609940563270 & 0.265 & 1.5011104170345925469 \\
\hline 0.070 & 1.0809622465945067573 & 0.270 & 1.5182816110001517220 \\
\hline 0.075 & 1.0876926219828829779 & 0.275 & 1.5359413824535917886 \\
\hline 0.080 & 1.0945669771981951837 & 0.280 & 1.5541084728730628870 \\
\hline 0.085 & 1.1015891348365177086 & 0.285 & 1.5728025442039259118 \\
\hline 0.090 & 1.1087630555865985256 & 0.290 & $1.5920 \quad 442334473745175$ \\
\hline 0.093 & 1.1160928440481103524 & 0.295 & 1.6118552110461658643 \\
\hline
\end{tabular}


TABLE 2 (continued)

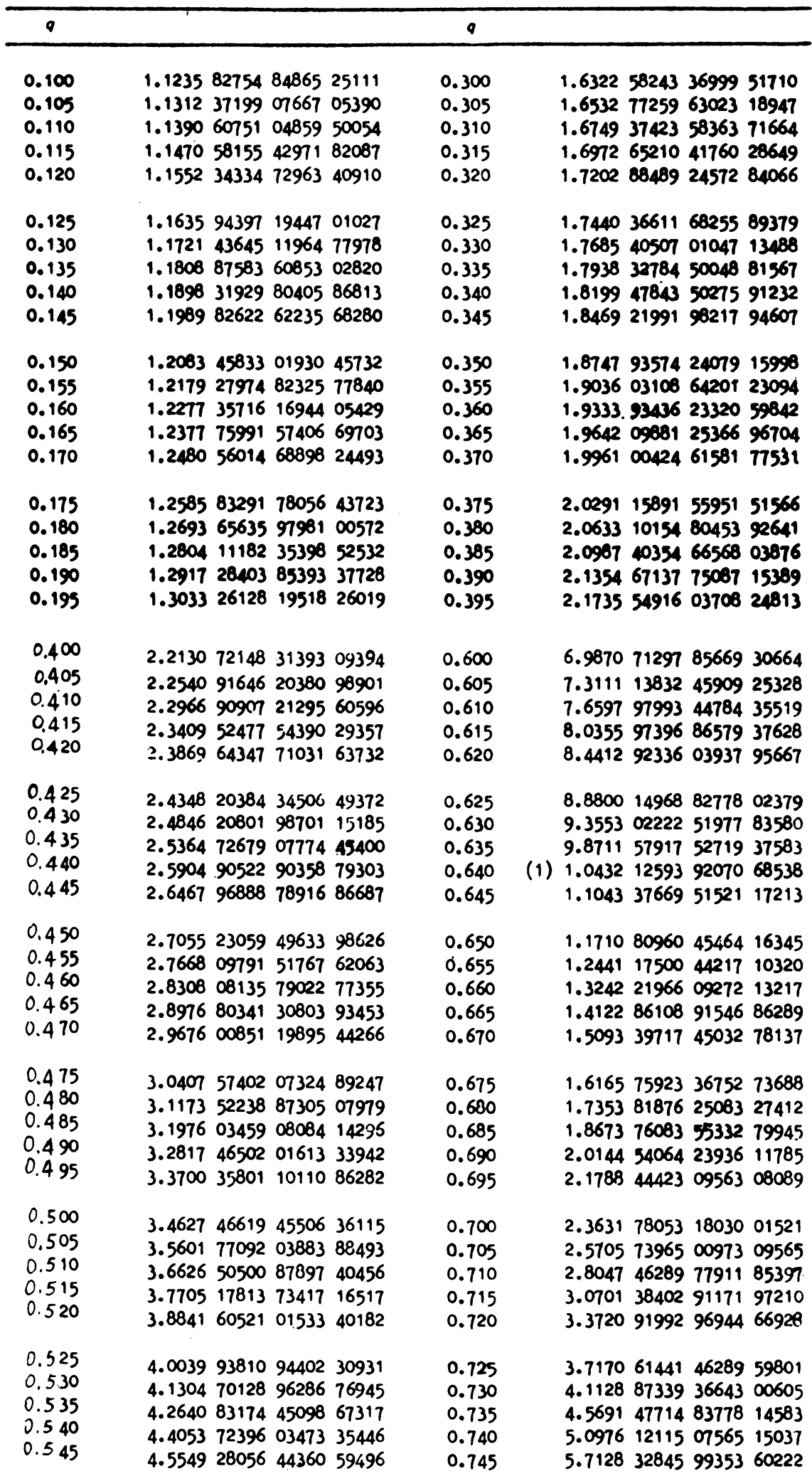


TABLE 2 (continued)

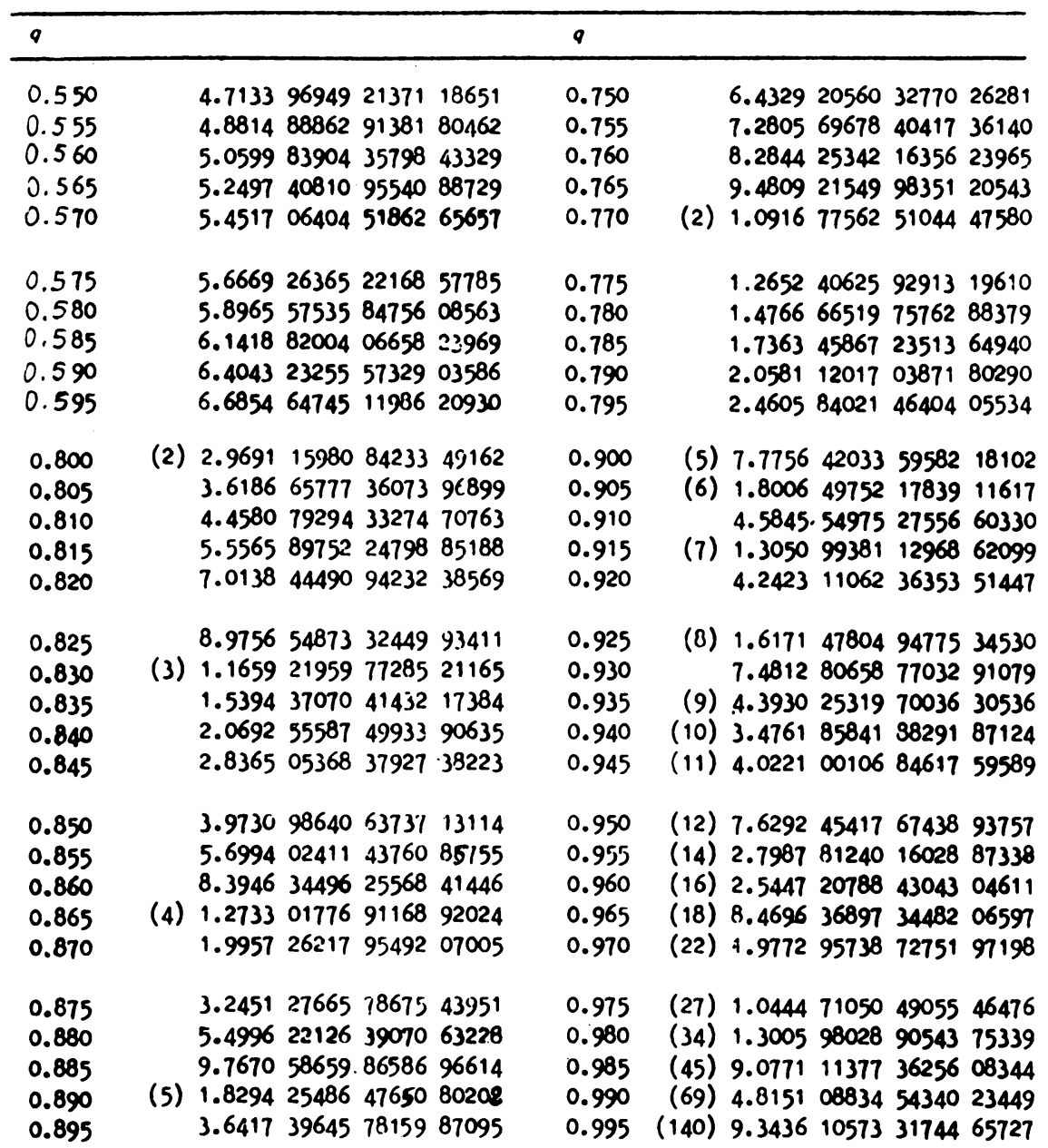

Institute of Numerical Analysis

University of Turin

Turin, Italy

Research and Development Group

Olivetti

Ivrea, Italy

1. L. J. SLATER, "Some new results on equivalent products," Proc. Cambridge Philos. Soc., v. 50, 1954, pp. 394-403.

2. L. J. SLATER, Generalized Hypergeometric Functions, Cambridge Univ. Press, Cambridge, 1966.

3. A. FLETCHER, J. C. P. MILLER, L. ROSENHEAD \& L. J. COMRIE, An Index of Mathematical Tables, 2nd ed., Addison-Wesley, Reading, Mass., 1962.

4. R. R. BUSH \& F. MOSTELLER, Stochastic Models for Learning, Wiley, New York, 1955.

5. L. GATTESCHI, "Procedimenti iterativi per il calcolo numerico di due prodotti infiniti,"

Rend. Sem. Mat. Univ. Politec. Torino, v. 29, 1969/70, pp. 187-201.

6. F. W. NEWMAN, Mathematical Tracts, Part II, Macmillan \& Bowes, Cambridge, 1889; rist. Bowes \& Bowes, Cambridge, 1912.

7. T. J. BROMWICH, An Introduction to the Theory of Infinite Series, Macmillan, New York, 1965.

8. M. L. BUZANO, "Sulle curve unite di talune trasformazioni puntuali," Rend. Sem. Mat. Univ. Politec. Torino, v. 25, 1965/66, pp. 193-210. 